\title{
Preparation and phytotoxicity evaluation of 11,13-dehydro seco-guaianolides
}

Nuria Chinchilla, ${ }^{\dagger}$ Alejandro Santana F., ${ }^{\dagger}$ Rosa M. Varela ${ }^{\dagger}$ Frank R. Fronczek,,+ José M. G. Molinillo, ${ }^{+}$and Francisco A. Maciast,**

$\dagger$ Allelopathy Group, Department of Organic Chemistry, School of Sciences, Institute of Biomolecules (INBIO), University of Cadiz, C/República Saharaui, s/n, 11510 Puerto Real (Cádiz), Spain.

* Department of Chemistry, Louisiana State University, Baton Rouge, LA 70803, U.S.A.

\section{Index}

Crystal structure of compound 12 by ORTEP diagram. S2 S7

${ }^{1} \mathrm{H}$ and ${ }^{13} \mathrm{C}$ spectra. S8 S14

Table S1. $\mathrm{IC}_{50}$ values compounds tested on coleoptile bioassay $\mathrm{S} 16$

Table S2. IC 50 values of compounds tested on STS bioassay S17 


\title{
Crystal structure of compound 12 by ORTEP diagram.
}

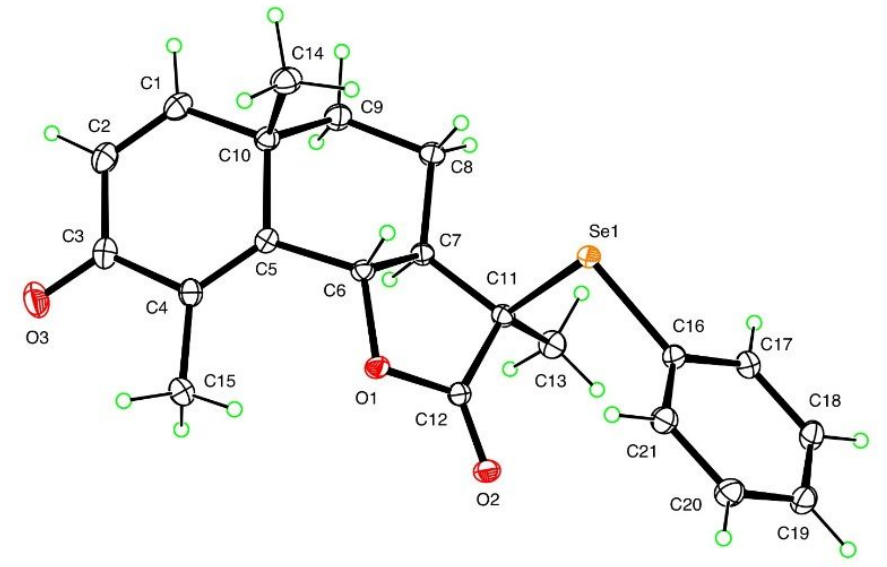

\section{Crystal data}

$\mathrm{C}_{21} \mathrm{H}_{22} \mathrm{O}_{3} \mathrm{Se}$

$M_{r}=401.35$

Orthorhombic, $P 2{ }_{1} 2_{1} 2_{1}$

Hall symbol: P 2ac $2 \mathrm{ab}$

$a=7.7213(3) \AA$

$b=10.2396(4) \AA$

$c=22.5926(8) \AA$

$V=1786.24(12) \AA^{3}$

$Z=4$

\author{
$F(000)=824$ \\ $D_{\mathrm{x}}=1.492 \mathrm{Mg} \mathrm{m}^{-3}$ \\ Mo $K \alpha$ radiation, $\lambda=0.71073 \AA$ \\ Cell parameters from 9859 reflections \\ $\theta=2.1-37.5^{\circ}$ \\ $\mu=2.12 \mathrm{~mm}^{-1}$ \\ $T=90 \mathrm{~K}$ \\ Fragment, Colourless \\ $0.27 \times 0.22 \times 0.19 \mathrm{~mm}$
}

\section{Data collection}

Bruker Kappa APEX-II DUO diffractometer

9258 independent reflections

Radiation source: fine-focus sealed tube

8764 reflections with $I>2 \sigma(I)$

TRIUMPH curved graphite

$R_{\text {int }}=0.024$

$\varphi$ and $\omega$ scans

$\theta_{\max }=37.5^{\circ}, \theta_{\min }=2.2^{\circ}$

Absorption correction: Multi-scan

SADABS (Sheldrick, 2004)

$h=-13 \rightarrow 13$

$T_{\min }=0.592, T_{\max }=0.650$

$k=-17 \rightarrow 17$

36325 measured reflections

$l=-38 \rightarrow 37$

\section{Refinement}

Refinement on $F^{2}$

Least-squares matrix: Full

$R\left[F^{2}>2 \sigma\left(F^{2}\right)\right]=0.019$
Secondary atom site location: Difference Fourier map

Hydrogen site location: Inferred from neighbouring sites

H-atom parameters constrained 

$w R\left(F^{2}\right)=0.043$
$w=1 /\left[\sigma^{2}\left(F_{\mathrm{o}}^{2}\right)\right]$
$S=1.00$
$(\Delta / \sigma)_{\max }=0.001$
9258 reflections
$\Delta \rho_{\max }=0.52$ e $\AA^{-3}$
229 parameters
$\Delta \rho_{\min }=-0.21$ e $\AA^{-3}$

0 restraints

Absolute structure: 4025 Friedel pairs (Flack, 1983)

Primary atom site location: Structure-invariant direct

methods

Flack parameter: $0.014(3)$

\section{Special details}

Geometry. All esds (except the esd in the dihedral angle between two 1.s. planes) are estimated using the full covariance matrix. The cell esds are taken into account individually in the estimation of esds in distances, angles and torsion angles; correlations between esds in cell parameters are only used when they are defined by crystal symmetry. An approximate (isotropic) treatment of cell esds is used for estimating esds involving l.s. planes.

Refinement. Refinement of $\mathrm{F}^{2}$ against ALL reflections. The weighted R-factor wR and goodness of fit $\mathrm{S}$ are based on $\mathrm{F}^{2}$, conventional Rfactors $R$ are based on $F$, with $F$ set to zero for negative $F^{2}$. The threshold expression of $F^{2}>2$ sigma( $\left.F^{2}\right)$ is used only for calculating Rfactors(gt) etc. and is not relevant to the choice of reflections for refinement. R-factors based on $\mathrm{F}^{2}$ are statistically about twice as large as those based on F, and R- factors based on ALL data will be even larger.

Fractional atomic coordinates and isotropic or equivalent isotropic displacement parameters $\left(A^{2}\right)$

\begin{tabular}{|c|c|c|c|c|}
\hline & $x$ & $y$ & $z$ & $U_{\text {iso }} * / U_{\text {eq }}$ \\
\hline Se1 & $0.578295(10)$ & $0.775789(7)$ & $0.817611(3)$ & $0.01243(2)$ \\
\hline $\mathrm{O} 1$ & $0.45522(7)$ & $0.62987(6)$ & $0.68599(2)$ & $0.01325(9)$ \\
\hline $\mathrm{O} 2$ & $0.30018(8)$ & $0.54112(6)$ & $0.75942(2)$ & $\begin{array}{l}0.01469 \\
(10)\end{array}$ \\
\hline $\mathrm{O} 3$ & $0.70936(10)$ & $0.51451(8)$ & $0.47679(3)$ & $\begin{array}{l}0.02482 \\
(14)\end{array}$ \\
\hline $\mathrm{C} 1$ & $1.00035(12)$ & $0.69260(8)$ & $0.56748(4)$ & $\begin{array}{l}0.01799 \\
(15)\end{array}$ \\
\hline H1 & 1.1162 & 0.7237 & 0.5659 & $0.022 *$ \\
\hline $\mathrm{C} 2$ & $0.94099(13)$ & $0.62479(9)$ & $0.52126(3)$ & $\begin{array}{l}0.01946 \\
(15)\end{array}$ \\
\hline $\mathrm{H} 2$ & 1.0155 & 0.6083 & 0.4886 & $0.023 *$ \\
\hline $\mathrm{C} 3$ & $0.76346(12)$ & $0.57551(8)$ & $0.51998(3)$ & $\begin{array}{l}0.01723 \\
(14)\end{array}$ \\
\hline $\mathrm{C} 4$ & $0.64914(11)$ & $0.60070(8)$ & $0.57186(3)$ & $\begin{array}{l}0.01426 \\
(13)\end{array}$ \\
\hline $\mathrm{C} 5$ & $0.71485(10)$ & $0.66240(7)$ & $0.61972(3)$ & $\begin{array}{l}0.01199 \\
(12)\end{array}$ \\
\hline C6 & $0.62799(9)$ & $0.68260(7)$ & 0.67877 (3) & $\begin{array}{l}0.01147 \\
(11)\end{array}$ \\
\hline H6 & 0.6245 & 0.7784 & 0.6874 & $0.014 *$ \\
\hline $\mathrm{C} 7$ & $0.73346(10)$ & $0.61516(7)$ & $0.72719(3)$ & $\begin{array}{l}0.01179 \\
(11)\end{array}$ \\
\hline $\mathrm{H} 7$ & 0.7581 & 0.5246 & 0.7129 & $0.014^{*}$ \\
\hline $\mathrm{C} 8$ & $0.90712(11)$ & $0.68219(9)$ & $0.73483(3)$ & $\begin{array}{l}0.01561 \\
(13)\end{array}$ \\
\hline H8A & 0.9751 & 0.6386 & 0.7663 & $0.019 *$ \\
\hline H8B & 0.8903 & 0.7748 & 0.7460 & $0.019^{*}$ \\
\hline
\end{tabular}




\begin{tabular}{|c|c|c|c|c|}
\hline C9 & $1.00233(10)$ & $0.67276(9)$ & $0.67541(3)$ & $\begin{array}{l}0.01678 \\
(14)\end{array}$ \\
\hline H9A & 1.0344 & 0.5804 & 0.6684 & $0.020^{*}$ \\
\hline H9B & 1.1109 & 0.7239 & 0.6780 & $0.020 *$ \\
\hline $\mathrm{C} 10$ & $0.89589(10)$ & $0.72264(8)$ & $0.62154(3)$ & $\begin{array}{l}0.01391 \\
(12)\end{array}$ \\
\hline C11 & $0.60209(10)$ & $0.60265(7)$ & $0.77726(3)$ & $\begin{array}{l}0.01182 \\
(12)\end{array}$ \\
\hline $\mathrm{C} 12$ & $0.43549(10)$ & $0.58591(7)$ & $0.74274(3)$ & $\begin{array}{l}0.01157 \\
(11)\end{array}$ \\
\hline C13 & $0.63564(11)$ & $0.49423(8)$ & $0.82151(4)$ & $\begin{array}{l}0.01633 \\
(13)\end{array}$ \\
\hline H13A & 0.5366 & 0.4867 & 0.8484 & $0.024 *$ \\
\hline H13B & 0.7403 & 0.5143 & 0.8443 & $0.024 *$ \\
\hline $\mathrm{H} 13 \mathrm{C}$ & 0.6517 & 0.4115 & 0.8004 & $0.024^{*}$ \\
\hline $\mathrm{C} 14$ & 0.88008 (12) & $0.87382(8)$ & $0.62355(4)$ & $\begin{array}{l}0.01861 \\
(15)\end{array}$ \\
\hline $\mathrm{H} 14 \mathrm{~A}$ & 0.9944 & 0.9130 & 0.6173 & $0.028 *$ \\
\hline H14B & 0.8351 & 0.9007 & 0.6622 & $0.028^{*}$ \\
\hline $\mathrm{H} 14 \mathrm{C}$ & 0.8008 & 0.9031 & 0.5924 & $0.028 *$ \\
\hline $\mathrm{C} 15$ & $0.46715(12)$ & $0.55135(9)$ & $0.56418(3)$ & $\begin{array}{l}0.01832 \\
(15)\end{array}$ \\
\hline $\mathrm{H} 15 \mathrm{~A}$ & 0.4653 & 0.4563 & 0.5693 & $0.027 *$ \\
\hline H15B & 0.4257 & 0.5734 & 0.5244 & $0.027^{*}$ \\
\hline $\mathrm{H} 15 \mathrm{C}$ & 0.3918 & 0.5921 & 0.5938 & $0.027^{*}$ \\
\hline C16 & $0.38854(10)$ & $0.73580(8)$ & 0.86898 (3) & $\begin{array}{l}0.01301 \\
(12)\end{array}$ \\
\hline C17 & $0.41964(13)$ & $0.68359(8)$ & $0.92510(3)$ & $\begin{array}{l}0.01591 \\
(12)\end{array}$ \\
\hline H17 & 0.5344 & 0.6642 & 0.9374 & $0.019 *$ \\
\hline $\mathrm{C} 18$ & $0.28030(13)$ & $0.66027(9)$ & $0.96277(3)$ & $\begin{array}{l}0.01857 \\
(15)\end{array}$ \\
\hline H18 & 0.3005 & 0.6249 & 1.0010 & $0.022 *$ \\
\hline C19 & $0.11253(12)$ & $0.68814(9)$ & $0.94505(3)$ & $\begin{array}{l}0.01894 \\
(15)\end{array}$ \\
\hline H19 & 0.0182 & 0.6709 & 0.9709 & $0.023 *$ \\
\hline $\mathrm{C} 20$ & $0.08249(12)$ & $0.74153(8)$ & $0.88923(3)$ & $0.01856(13)$ \\
\hline $\mathrm{H} 20$ & -0.0323 & 0.7618 & 0.8772 & $0.022^{*}$ \\
\hline $\mathrm{C} 21$ & $0.22041(11)$ & $0.76508(8)$ & $0.85128(3)$ & $0.01527(12)$ \\
\hline $\mathrm{H} 21$ & 0.1999 & 0.8012 & 0.8132 & $0.018^{*}$ \\
\hline
\end{tabular}

Atomic displacement parameters $\left(A^{2}\right)$

$\begin{array}{lllllll} & U_{11} & U_{22} & U_{33} & U_{12} & U_{13} & U_{23} \\ \text { Se1 } & 0.01338(3) & 0.01178(3) & 0.01213(3) & -0.00254(3) & 0.00133(3) & -0.00109(2) \\ \text { O1 } & 0.0091(2) & 0.0183(2) & 0.01231(19) & -0.00129(17) & -0.00037(18) & 0.00135(18) \\ \text { O2 } & 0.0120(3) & 0.0152(2) & 0.0169(2) & -0.0023(2) & 0.00154(18) & -0.00102(19) \\ \text { O3 } & 0.0273(4) & 0.0316(4) & 0.0155(2) & -0.0005(3) & -0.0002(2) & -0.0075(2) \\ \text { C1 } & 0.0154(4) & 0.0195(4) & 0.0190(3) & 0.0005(3) & 0.0047(3) & 0.0007(3) \\ \text { C2 } & 0.0199(4) & 0.0219(4) & 0.0165(3) & 0.0015(3) & 0.0050(3) & -0.0014(3)\end{array}$




$\begin{array}{lllllll}\text { C3 } & 0.0210(4) & 0.0174(3) & 0.0134(3) & 0.0027(3) & 0.0012(3) & -0.0001(2) \\ \text { C4 } & 0.0161(3) & 0.0145(3) & 0.0121(3) & 0.0019(3) & -0.0007(2) & 0.0008(2) \\ \text { C5 } & 0.0119(3) & 0.0120(3) & 0.0121(2) & 0.0018(2) & 0.0007(2) & 0.0011(2) \\ \text { C6 } & 0.0091(3) & 0.0128(3) & 0.0125(2) & -0.0002(2) & -0.0001(2) & 0.0005(2) \\ \text { C7 } & 0.0094(3) & 0.0136(3) & 0.0125(3) & 0.0008(2) & -0.0006(2) & -0.0003(2) \\ \text { C8 } & 0.0097(3) & 0.0224(3) & 0.0147(3) & -0.0011(3) & -0.0014(2) & -0.0004(2) \\ \text { C9 } & 0.0109(3) & 0.0222(3) & 0.0172(3) & 0.0011(3) & 0.0002(2) & 0.0001(3) \\ \text { C10 } & 0.0126(3) & 0.0145(3) & 0.0146(2) & 0.0001(3) & 0.0013(2) & -0.0003(2) \\ \text { C11 } & 0.0115(3) & 0.0121(3) & 0.0118(2) & -0.0001(2) & -0.0010(2) & 0.0001(2) \\ \text { C12 } & 0.0115(3) & 0.0110(3) & 0.0122(2) & 0.0004(2) & 0.0005(2) & -0.0006(2) \\ \text { C13 } & 0.0184(3) & 0.0147(3) & 0.0159(3) & 0.0010(3) & -0.0020(3) & 0.0033(3) \\ \text { C14 } & 0.0189(4) & 0.0149(3) & 0.0220(3) & -0.0017(3) & 0.0032(3) & -0.0001(3) \\ \text { C15 } & 0.0183(4) & 0.0226(4) & 0.0140(3) & -0.0026(3) & -0.0026(2) & -0.0004(3) \\ \text { C16 } & 0.0142(3) & 0.0128(3) & 0.0120(2) & -0.0016(2) & 0.0013(2) & -0.0012(2) \\ \text { C17 } & 0.0172(3) & 0.0180(3) & 0.0125(2) & -0.0015(3) & -0.0011(3) & -0.0007(2) \\ \text { C18 } & 0.0229(4) & 0.0204(4) & 0.0124(3) & -0.0028(3) & 0.0025(3) & 0.0000(2) \\ \text { C19 } & 0.0195(4) & 0.0191(3) & 0.0182(3) & -0.0028(3) & 0.0063(3) & -0.0039(3) \\ \text { C20 } & 0.0147(3) & 0.0202(3) & 0.0208(3) & -0.0001(3) & 0.0021(3) & -0.0021(2) \\ \text { C21 } & 0.0148(3) & 0.0153(3) & 0.0157(3) & 0.0004(3) & 0.0003(2) & -0.0005(2)\end{array}$

Geometric parameters $\left(A,{ }^{\circ}\right)$

\begin{tabular}{|c|c|c|c|}
\hline $\mathrm{Se} 1-\mathrm{C} 16$ & $1.9135(7)$ & C9-H9A & 0.9900 \\
\hline $\mathrm{Se} 1-\mathrm{C} 11$ & $2.0020(7)$ & C9-H9B & 0.9900 \\
\hline $\mathrm{O} 1-\mathrm{C} 12$ & $1.3674(8)$ & $\mathrm{C} 10-\mathrm{C} 14$ & $1.5536(13)$ \\
\hline $\mathrm{O} 1-\mathrm{C} 6$ & $1.4484(9)$ & $\mathrm{C} 11-\mathrm{C} 12$ & $1.5141(11)$ \\
\hline $\mathrm{O} 2-\mathrm{C} 12$ & $1.2015(10)$ & $\mathrm{C} 11-\mathrm{C} 13$ & $1.5163(11)$ \\
\hline $\mathrm{O} 3-\mathrm{C} 3$ & $1.2316(10)$ & $\mathrm{C} 13-\mathrm{H} 13 \mathrm{~A}$ & 0.9800 \\
\hline $\mathrm{C} 1-\mathrm{C} 2$ & $1.3351(12)$ & C13-H13B & 0.9800 \\
\hline $\mathrm{C} 1-\mathrm{C} 10$ & $1.4956(11)$ & $\mathrm{C} 13-\mathrm{H} 13 \mathrm{C}$ & 0.9800 \\
\hline $\mathrm{C} 1-\mathrm{H} 1$ & 0.9500 & $\mathrm{C} 14-\mathrm{H} 14 \mathrm{~A}$ & 0.9800 \\
\hline $\mathrm{C} 2-\mathrm{C} 3$ & $1.4610(14)$ & $\mathrm{C} 14-\mathrm{H} 14 \mathrm{~B}$ & 0.9800 \\
\hline $\mathrm{C} 2-\mathrm{H} 2$ & 0.9500 & $\mathrm{C} 14-\mathrm{H} 14 \mathrm{C}$ & 0.9800 \\
\hline $\mathrm{C} 3-\mathrm{C} 4$ & $1.4898(11)$ & $\mathrm{C} 15-\mathrm{H} 15 \mathrm{~A}$ & 0.9800 \\
\hline $\mathrm{C} 4-\mathrm{C} 5$ & $1.3511(11)$ & $\mathrm{C} 15-\mathrm{H} 15 \mathrm{~B}$ & 0.9800 \\
\hline $\mathrm{C} 4-\mathrm{C} 15$ & $1.5034(13)$ & $\mathrm{C} 15-\mathrm{H} 15 \mathrm{C}$ & 0.9800 \\
\hline $\mathrm{C} 5-\mathrm{C} 6$ & $1.5075(10)$ & $\mathrm{C} 16-\mathrm{C} 21$ & $\begin{array}{l}1.3911 \\
(11)\end{array}$ \\
\hline $\mathrm{C} 5-\mathrm{C} 10$ & $1.5285(11)$ & $\mathrm{C} 16-\mathrm{C} 17$ & $\begin{array}{l}1.3967 \\
(10)\end{array}$ \\
\hline $\mathrm{C} 6-\mathrm{C} 7$ & $1.5286(10)$ & $\mathrm{C} 17-\mathrm{C} 18$ & $\begin{array}{l}1.3925 \\
(12)\end{array}$ \\
\hline $\mathrm{C} 6-\mathrm{H} 6$ & 1.0000 & $\mathrm{C} 17-\mathrm{H} 17$ & 0.9500 \\
\hline
\end{tabular}




\begin{tabular}{|c|c|c|c|}
\hline $\mathrm{C} 7-\mathrm{C} 8$ & $1.5162(12)$ & $\mathrm{C} 18-\mathrm{C} 19$ & $\begin{array}{l}1.3856 \\
(14)\end{array}$ \\
\hline $\mathrm{C} 7-\mathrm{C} 11$ & $1.5249(11)$ & $\mathrm{C} 18-\mathrm{H} 18$ & 0.9500 \\
\hline $\mathrm{C} 7-\mathrm{H} 7$ & 1.0000 & $\mathrm{C} 19-\mathrm{C} 20$ & $\begin{array}{l}1.3940 \\
(12)\end{array}$ \\
\hline $\mathrm{C} 8-\mathrm{C} 9$ & $1.5336(11)$ & $\mathrm{C} 19-\mathrm{H} 19$ & 0.9500 \\
\hline $\mathrm{C} 8-\mathrm{H} 8 \mathrm{~A}$ & 0.9900 & $\mathrm{C} 20-\mathrm{C} 21$ & $\begin{array}{l}1.3882 \\
(12)\end{array}$ \\
\hline $\mathrm{C} 8-\mathrm{H} 8 \mathrm{~B}$ & 0.9900 & $\mathrm{C} 20-\mathrm{H} 20$ & 0.9500 \\
\hline $\mathrm{C} 9-\mathrm{C} 10$ & $1.5549(11)$ & $\mathrm{C} 21-\mathrm{H} 21$ & 0.9500 \\
\hline $\mathrm{C} 16-\mathrm{Se} 1-\mathrm{C} 11$ & $99.03(3)$ & $\mathrm{C} 14-\mathrm{C} 10-\mathrm{C} 9$ & $110.24(7)$ \\
\hline $\mathrm{C} 12-\mathrm{O} 1-\mathrm{C} 6$ & $109.33(6)$ & $\mathrm{C} 12-\mathrm{C} 11-\mathrm{C} 13$ & $113.70(6)$ \\
\hline $\mathrm{C} 2-\mathrm{C} 1-\mathrm{C} 10$ & $124.09(8)$ & $\mathrm{C} 12-\mathrm{C} 11-\mathrm{C} 7$ & $101.10(5)$ \\
\hline $\mathrm{C} 2-\mathrm{C} 1-\mathrm{H} 1$ & 118.0 & $\mathrm{C} 13-\mathrm{C} 11-\mathrm{C} 7$ & $115.92(7)$ \\
\hline $\mathrm{C} 10-\mathrm{C} 1-\mathrm{H} 1$ & 118.0 & $\mathrm{C} 12-\mathrm{C} 11-\mathrm{Se} 1$ & $104.88(5)$ \\
\hline $\mathrm{C} 1-\mathrm{C} 2-\mathrm{C} 3$ & $121.14(7)$ & $\mathrm{C} 13-\mathrm{C} 11-\mathrm{Se} 1$ & $111.34(5)$ \\
\hline $\mathrm{C} 1-\mathrm{C} 2-\mathrm{H} 2$ & 119.4 & $\mathrm{C} 7-\mathrm{C} 11-\mathrm{Se} 1$ & $108.93(5)$ \\
\hline $\mathrm{C} 3-\mathrm{C} 2-\mathrm{H} 2$ & 119.4 & $\mathrm{O} 2-\mathrm{C} 12-\mathrm{O} 1$ & $121.09(7)$ \\
\hline $\mathrm{O} 3-\mathrm{C} 3-\mathrm{C} 2$ & $120.60(7)$ & $\mathrm{O} 2-\mathrm{C} 12-\mathrm{C} 11$ & $128.35(6)$ \\
\hline $\mathrm{O} 3-\mathrm{C} 3-\mathrm{C} 4$ & $120.68(8)$ & $\mathrm{O} 1-\mathrm{C} 12-\mathrm{C} 11$ & $110.55(6)$ \\
\hline $\mathrm{C} 2-\mathrm{C} 3-\mathrm{C} 4$ & $118.72(7)$ & $\mathrm{C} 11-\mathrm{C} 13-\mathrm{H} 13 \mathrm{~A}$ & 109.5 \\
\hline $\mathrm{C} 5-\mathrm{C} 4-\mathrm{C} 3$ & $119.22(8)$ & $\mathrm{C} 11-\mathrm{C} 13-\mathrm{H} 13 \mathrm{~B}$ & 109.5 \\
\hline $\mathrm{C} 5-\mathrm{C} 4-\mathrm{C} 15$ & $126.90(7)$ & $\mathrm{H} 13 \mathrm{~A}-\mathrm{C} 13-\mathrm{H} 13 \mathrm{~B}$ & 109.5 \\
\hline $\mathrm{C} 3-\mathrm{C} 4-\mathrm{C} 15$ & $113.88(7)$ & $\mathrm{C} 11-\mathrm{C} 13-\mathrm{H} 13 \mathrm{C}$ & 109.5 \\
\hline $\mathrm{C} 4-\mathrm{C} 5-\mathrm{C} 6$ & $127.25(7)$ & $\mathrm{H} 13 \mathrm{~A}-\mathrm{C} 13-\mathrm{H} 13 \mathrm{C}$ & 109.5 \\
\hline $\mathrm{C} 4-\mathrm{C} 5-\mathrm{C} 10$ & $123.62(7)$ & $\mathrm{H} 13 \mathrm{~B}-\mathrm{C} 13-\mathrm{H} 13 \mathrm{C}$ & 109.5 \\
\hline $\mathrm{C} 6-\mathrm{C} 5-\mathrm{C} 10$ & $109.12(6)$ & $\mathrm{C} 10-\mathrm{C} 14-\mathrm{H} 14 \mathrm{~A}$ & 109.5 \\
\hline $\mathrm{O} 1-\mathrm{C} 6-\mathrm{C} 5$ & $117.28(6)$ & $\mathrm{C} 10-\mathrm{C} 14-\mathrm{H} 14 \mathrm{~B}$ & 109.5 \\
\hline $\mathrm{O} 1-\mathrm{C} 6-\mathrm{C} 7$ & $103.98(6)$ & $\mathrm{H} 14 \mathrm{~A}-\mathrm{C} 14-\mathrm{H} 14 \mathrm{~B}$ & 109.5 \\
\hline $\mathrm{C} 5-\mathrm{C} 6-\mathrm{C} 7$ & $109.53(6)$ & $\mathrm{C} 10-\mathrm{C} 14-\mathrm{H} 14 \mathrm{C}$ & 109.5 \\
\hline $\mathrm{O} 1-\mathrm{C} 6-\mathrm{H} 6$ & 108.6 & $\mathrm{H} 14 \mathrm{~A}-\mathrm{C} 14-\mathrm{H} 14 \mathrm{C}$ & 109.5 \\
\hline $\mathrm{C} 5-\mathrm{C} 6-\mathrm{H} 6$ & 108.6 & $\mathrm{H} 14 \mathrm{~B}-\mathrm{C} 14-\mathrm{H} 14 \mathrm{C}$ & 109.5 \\
\hline $\mathrm{C} 7-\mathrm{C} 6-\mathrm{H} 6$ & 108.6 & $\mathrm{C} 4-\mathrm{C} 15-\mathrm{H} 15 \mathrm{~A}$ & 109.5 \\
\hline $\mathrm{C} 8-\mathrm{C} 7-\mathrm{C} 11$ & $122.80(6)$ & $\mathrm{C} 4-\mathrm{C} 15-\mathrm{H} 15 \mathrm{~B}$ & 109.5 \\
\hline $\mathrm{C} 8-\mathrm{C} 7-\mathrm{C} 6$ & $110.37(6)$ & $\mathrm{H} 15 \mathrm{~A}-\mathrm{C} 15-\mathrm{H} 15 \mathrm{~B}$ & 109.5 \\
\hline $\mathrm{C} 11-\mathrm{C} 7-\mathrm{C} 6$ & $102.39(6)$ & $\mathrm{C} 4-\mathrm{C} 15-\mathrm{H} 15 \mathrm{C}$ & 109.5 \\
\hline $\mathrm{C} 8-\mathrm{C} 7-\mathrm{H} 7$ & 106.8 & $\mathrm{H} 15 \mathrm{~A}-\mathrm{C} 15-\mathrm{H} 15 \mathrm{C}$ & 109.5 \\
\hline $\mathrm{C} 11-\mathrm{C} 7-\mathrm{H} 7$ & 106.8 & $\mathrm{H} 15 \mathrm{~B}-\mathrm{C} 15-\mathrm{H} 15 \mathrm{C}$ & 109.5 \\
\hline $\mathrm{C} 6-\mathrm{C} 7-\mathrm{H} 7$ & 106.8 & $\mathrm{C} 21-\mathrm{C} 16-\mathrm{C} 17$ & $120.25(7)$ \\
\hline $\mathrm{C} 7-\mathrm{C} 8-\mathrm{C} 9$ & $107.20(6)$ & $\mathrm{C} 21-\mathrm{C} 16-\mathrm{Se} 1$ & $119.61(5)$ \\
\hline $\mathrm{C} 7-\mathrm{C} 8-\mathrm{H} 8 \mathrm{~A}$ & 110.3 & $\mathrm{C} 17-\mathrm{C} 16-\mathrm{Se} 1$ & $120.06(6)$ \\
\hline $\mathrm{C} 9-\mathrm{C} 8-\mathrm{H} 8 \mathrm{~A}$ & 110.3 & $\mathrm{C} 18-\mathrm{C} 17-\mathrm{C} 16$ & $119.19(8)$ \\
\hline $\mathrm{C} 7-\mathrm{C} 8-\mathrm{H} 8 \mathrm{~B}$ & 110.3 & $\mathrm{C} 18-\mathrm{C} 17-\mathrm{H} 17$ & 120.4 \\
\hline $\mathrm{C} 9-\mathrm{C} 8-\mathrm{H} 8 \mathrm{~B}$ & 110.3 & $\mathrm{C} 16-\mathrm{C} 17-\mathrm{H} 17$ & 120.4 \\
\hline $\mathrm{H} 8 \mathrm{~A}-\mathrm{C} 8-\mathrm{H} 8 \mathrm{~B}$ & 108.5 & $\mathrm{C} 19-\mathrm{C} 18-\mathrm{C} 17$ & $120.69(7)$ \\
\hline $\mathrm{C} 8-\mathrm{C} 9-\mathrm{C} 10$ & $114.28(6)$ & $\mathrm{C} 19-\mathrm{C} 18-\mathrm{H} 18$ & 119.7 \\
\hline $\mathrm{C} 8-\mathrm{C} 9-\mathrm{H} 9 \mathrm{~A}$ & 108.7 & $\mathrm{C} 17-\mathrm{C} 18-\mathrm{H} 18$ & 119.7 \\
\hline $\mathrm{C} 10-\mathrm{C} 9-\mathrm{H} 9 \mathrm{~A}$ & 108.7 & $\mathrm{C} 18-\mathrm{C} 19-\mathrm{C} 20$ & $119.85(8)$ \\
\hline $\mathrm{C} 8-\mathrm{C} 9-\mathrm{H} 9 \mathrm{~B}$ & 108.7 & $\mathrm{C} 18-\mathrm{C} 19-\mathrm{H} 19$ & 120.1 \\
\hline $\mathrm{C} 10-\mathrm{C} 9-\mathrm{H} 9 \mathrm{~B}$ & 108.7 & $\mathrm{C} 20-\mathrm{C} 19-\mathrm{H} 19$ & 120.1 \\
\hline
\end{tabular}




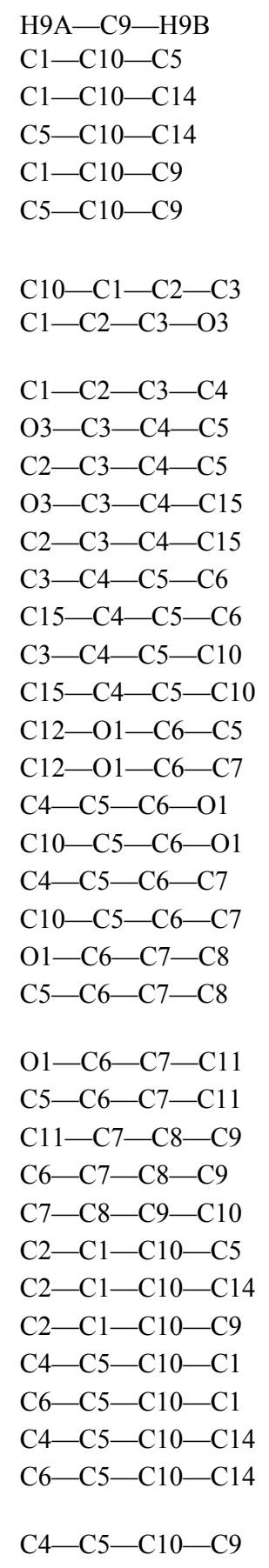

107.6

112.84 (6)

$105.73(7)$

109.33 (6)

106.66 (6)

111.84 (6)

0.96 (13)

$179.06(9)$

$-0.82(12)$

$176.83(8)$

-3.29 (12)

-2.82 (11)

$177.06(7)$

$-172.13(7)$

7.48 (13)

$7.24(12)$

$-173.16(8)$

144.48 (6)

$23.41(7)$

0.47 (11)

-178.98 (6)

$118.59(8)$

$-60.85(8)$

-167.00 (6)

$66.88(8)$

$-34.68(7)$

$-160.80(6)$

$179.07(7)$

$-60.17(8)$

52.55 (9)

2.45 (12)

-117.01 (9)

125.63 (9)

$-6.76(11)$

$172.71(7)$

$110.61(8)$

$-69.93(7)$

$-127.01(8)$
$\begin{array}{ll}\mathrm{C} 21-\mathrm{C} 20-\mathrm{C} 19 & 119.95(8) \\ \mathrm{C} 21-\mathrm{C} 20-\mathrm{H} 20 & 120.0 \\ \mathrm{C} 19-\mathrm{C} 20-\mathrm{H} 20 & 120.0 \\ \mathrm{C} 20-\mathrm{C} 21-\mathrm{C} 16 & 120.06(7) \\ \mathrm{C} 20-\mathrm{C} 21-\mathrm{H} 21 & 120.0 \\ \mathrm{C} 16-\mathrm{C} 21-\mathrm{H} 21 & 120.0\end{array}$

$\mathrm{C} 6-\mathrm{C} 5-\mathrm{C} 10-\mathrm{C} 9 \quad 52.45(8)$

$\mathrm{C} 8-\mathrm{C} 9-\mathrm{C} 10-\mathrm{C} 1 \quad-173.95$

(7)

$\mathrm{C} 8-\mathrm{C} 9-\mathrm{C} 10-\mathrm{C} 5 \quad-50.14(9)$

$\mathrm{C} 8-\mathrm{C} 9-\mathrm{C} 10-\mathrm{C} 14 \quad 71.72(9)$

$\mathrm{C} 8-\mathrm{C} 7-\mathrm{C} 11-\mathrm{C} 12 \quad 156.60(7)$

$\mathrm{C} 6-\mathrm{C} 7-\mathrm{C} 11-\mathrm{C} 12$

$\mathrm{C} 8-\mathrm{C} 7-\mathrm{C} 11-\mathrm{C} 13 \quad-79.99(9)$

$\mathrm{C} 6-\mathrm{C} 7-\mathrm{C} 11-\mathrm{C} 13 \quad 155.57$ (6)

$\mathrm{C} 8-\mathrm{C} 7-\mathrm{C} 11-\mathrm{Se} 1 \quad 46.49(8)$

$\mathrm{C} 6-\mathrm{C} 7-\mathrm{C} 11-\mathrm{Se} 1 \quad-77.96(6)$

$\mathrm{C} 16-\mathrm{Se} 1-\mathrm{C} 11-\mathrm{C} 12$

$\mathrm{C} 16-\mathrm{Se} 1-\mathrm{C} 11-\mathrm{C} 13 \quad-58.33$ (6)

$\mathrm{C} 16-\mathrm{Se} 1-\mathrm{C} 11-\mathrm{C} 7 \quad 172.61(5)$

$\mathrm{C} 6-\mathrm{O} 1-\mathrm{C} 12-\mathrm{O} 2 \quad 177.96(7)$

$\mathrm{C} 6-\mathrm{O} 1-\mathrm{C} 12-\mathrm{C} 11 \quad-2.23(8)$

$\mathrm{C} 13-\mathrm{C} 11-\mathrm{C} 12-\mathrm{O} 2 \quad 35.17(11)$

$\mathrm{C} 7-\mathrm{C} 11-\mathrm{C} 12-\mathrm{O} 2 \quad 160.09(8)$

$\mathrm{Se} 1-\mathrm{C} 11-\mathrm{C} 12-\mathrm{O} 2 \quad-86.68(8)$

$\mathrm{C} 13-\mathrm{C} 11-\mathrm{C} 12-\mathrm{O} 1 \quad-144.63$

$\mathrm{C} 7-\mathrm{C} 11-\mathrm{C} 12-\mathrm{O} 1 \quad-19.70(7)$

$\mathrm{Se} 1-\mathrm{C} 11-\mathrm{C} 12-\mathrm{O} 1 \quad 93.52(6)$

$\mathrm{C} 11-\mathrm{Se} 1-\mathrm{C} 16-\mathrm{C} 21 \quad-94.55$ (7)

$\mathrm{C} 11-\mathrm{Se} 1-\mathrm{C} 16-\mathrm{C} 17 \quad 88.66(6)$

$\mathrm{C} 21-\mathrm{C} 16-\mathrm{C} 17-\mathrm{C} 18 \quad 0.55$ (12)

$\mathrm{Se} 1-\mathrm{C} 16-\mathrm{C} 17-\mathrm{C} 18 \quad 177.33$ (6)

$\mathrm{C} 16-\mathrm{C} 17-\mathrm{C} 18-\mathrm{C} 19 \quad 0.08(12)$

$\mathrm{C} 17-\mathrm{C} 18-\mathrm{C} 19-\mathrm{C} 20 \quad-0.76$ (13)

$\mathrm{C} 18-\mathrm{C} 19-\mathrm{C} 20-\mathrm{C} 21 \quad 0.81(13)$

$\mathrm{C} 19-\mathrm{C} 20-\mathrm{C} 21-\mathrm{C} 16 \quad-0.19$ (12)

$\mathrm{C} 17-\mathrm{C} 16-\mathrm{C} 21-\mathrm{C} 20 \quad-0.50$ (12)

$\mathrm{Se} 1-\mathrm{C} 16-\mathrm{C} 21-\mathrm{C} 20$

$-177.29$

(6) 


\section{${ }^{1} \mathrm{H}-\mathrm{NMR}$ and ${ }^{13} \mathrm{C}-\mathrm{NMR}$ Spectroscopy}

4-methyl-5-((2S, 3S)-4-methylene-5-oxo-3-(3-oxobutyl)tetrahydrofuran-2-yl)cyclopent-4ene-1,3-dione (10).

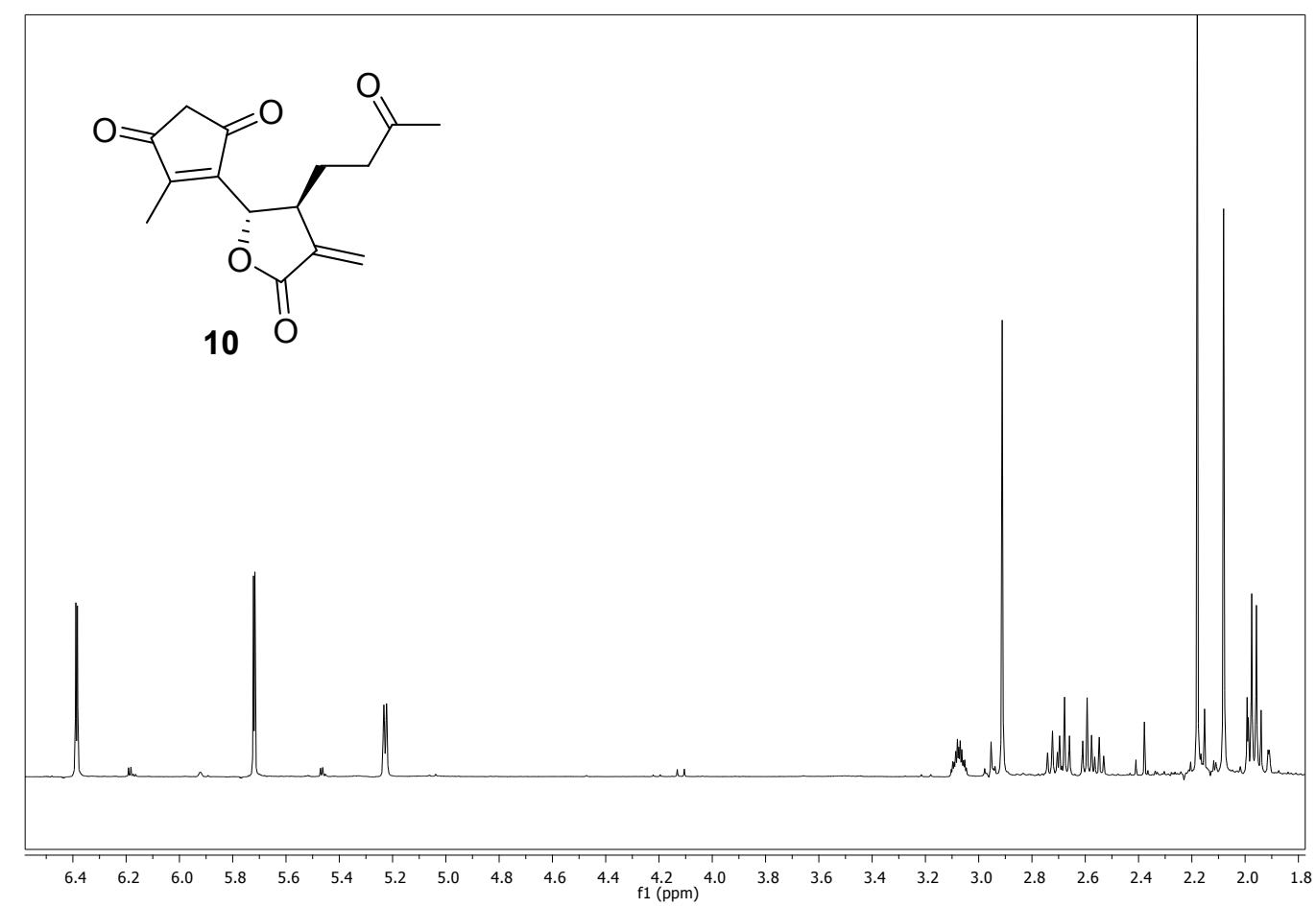




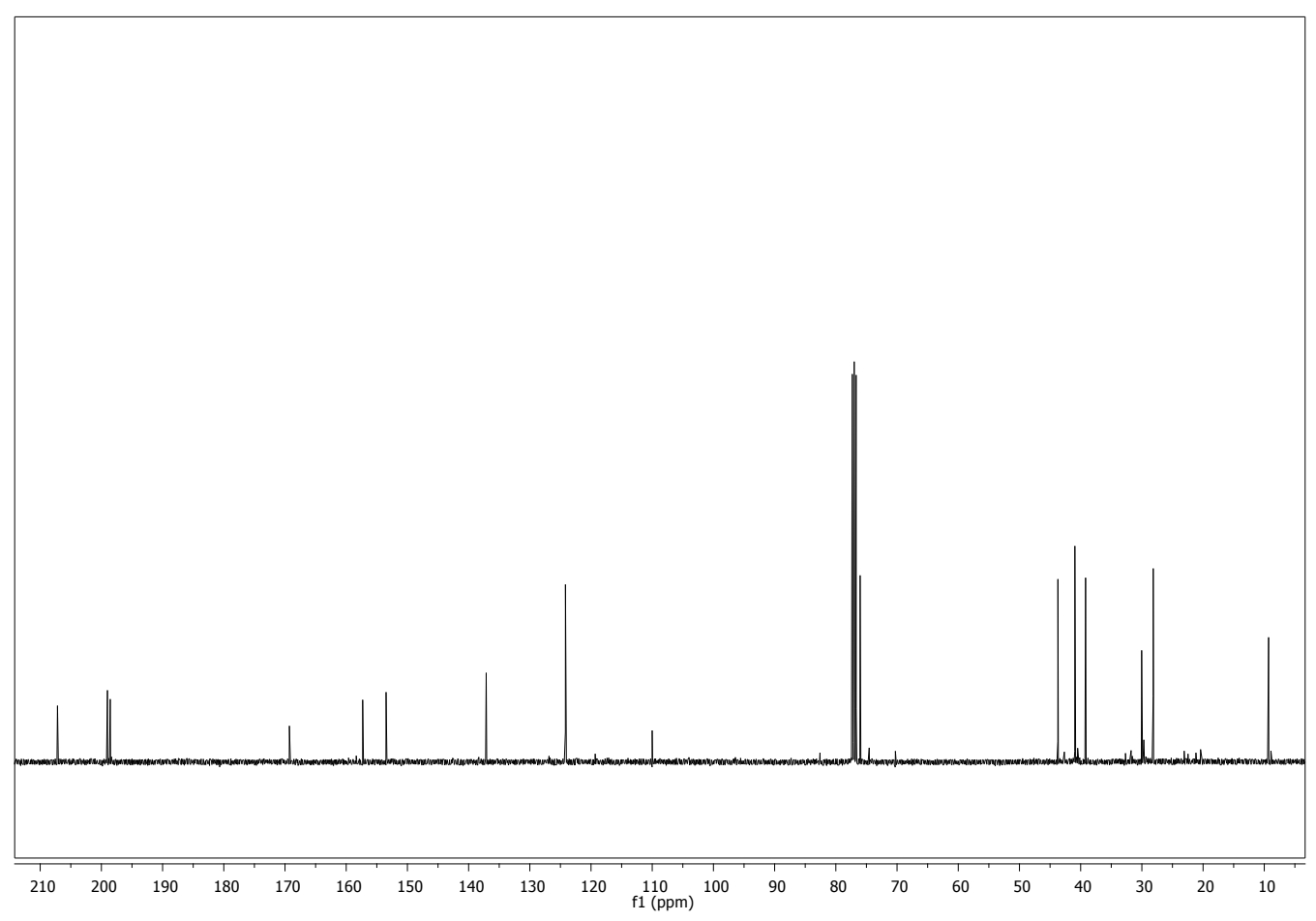

(3S,3aR,5aS,9bS)-3,5a,9-trimethyl-3-(phenylselanyl)-3a,4,5,5a-tetrahydronaphtho[1,2-b]-furan2,8(3H,9bH)-dione (12).

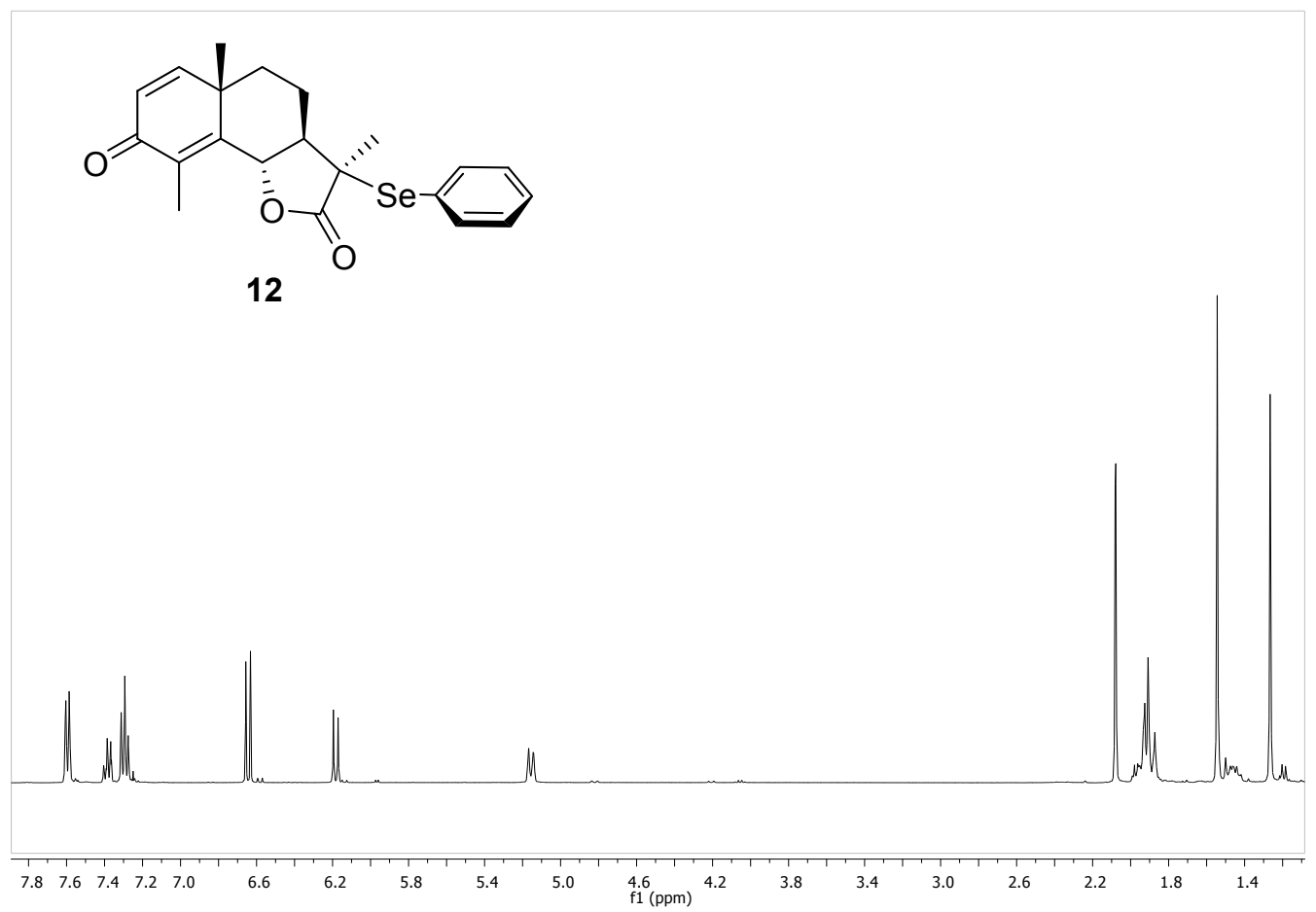




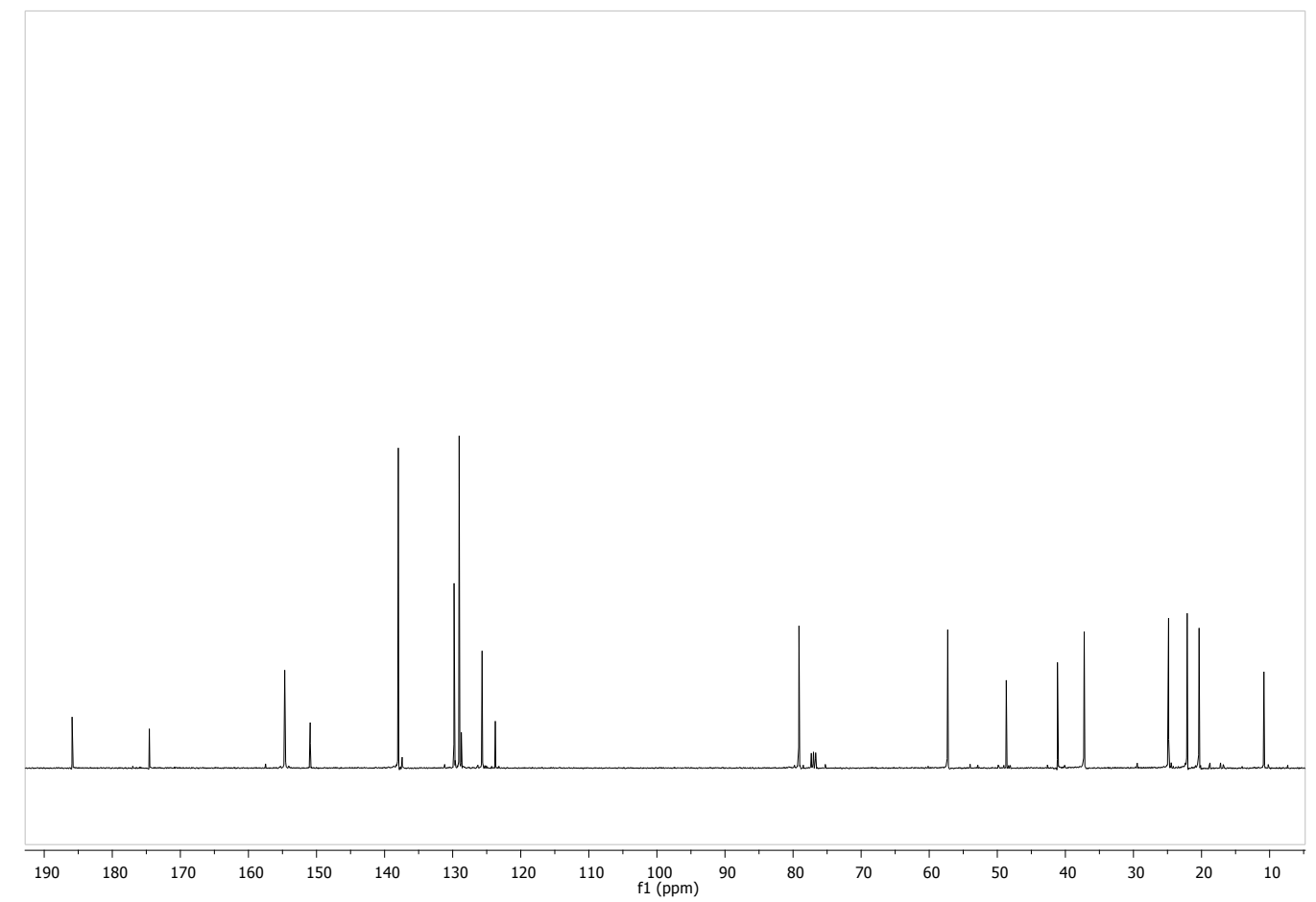



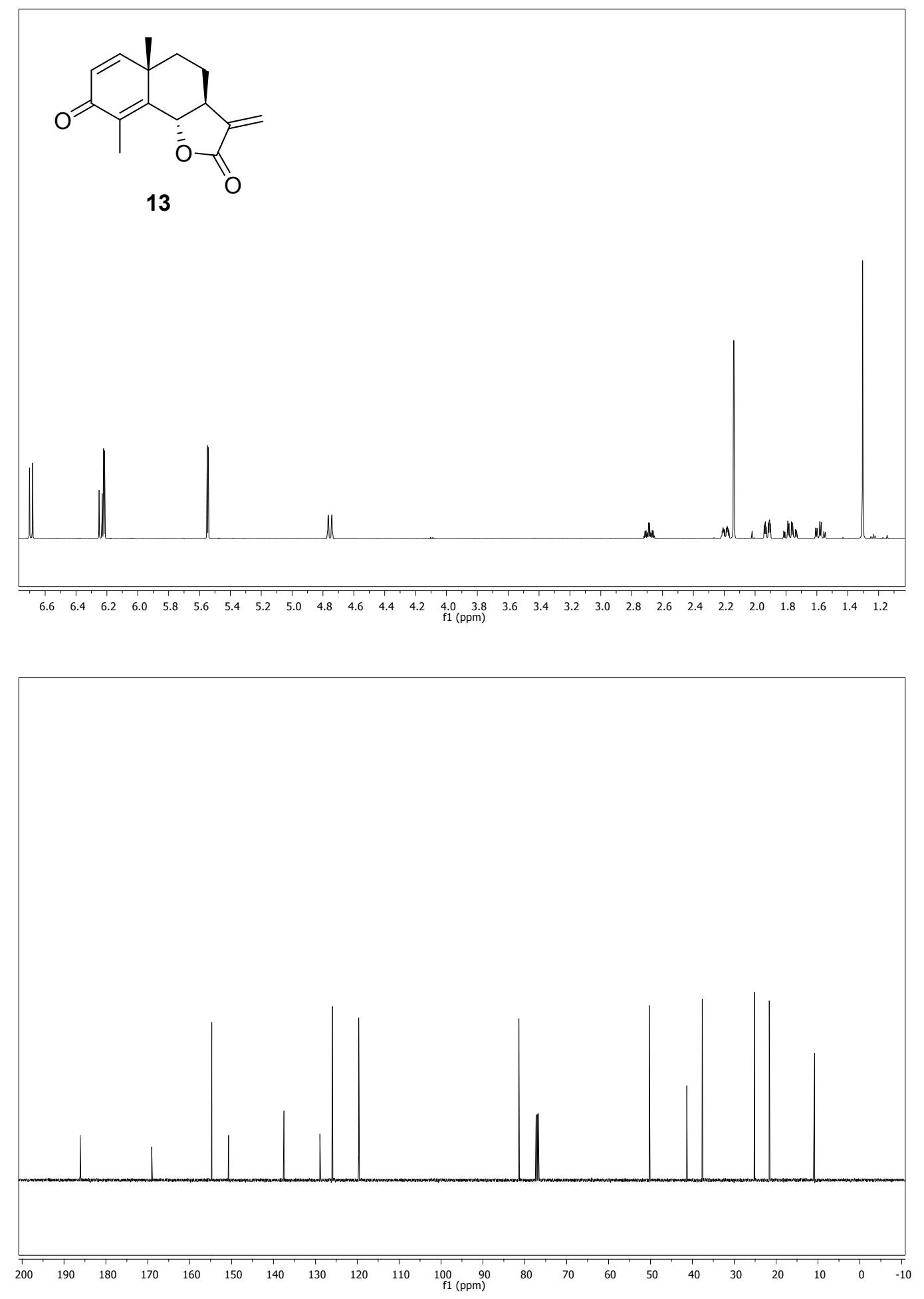
(3aS,6R,9bS)-6-hydroxy-6,9-dimethyl-3-methylene-3a,4,5,6,6a, 7-hexahydroazuleno[4,5-b]furan-2,8(3H,9bH)-dione (14).
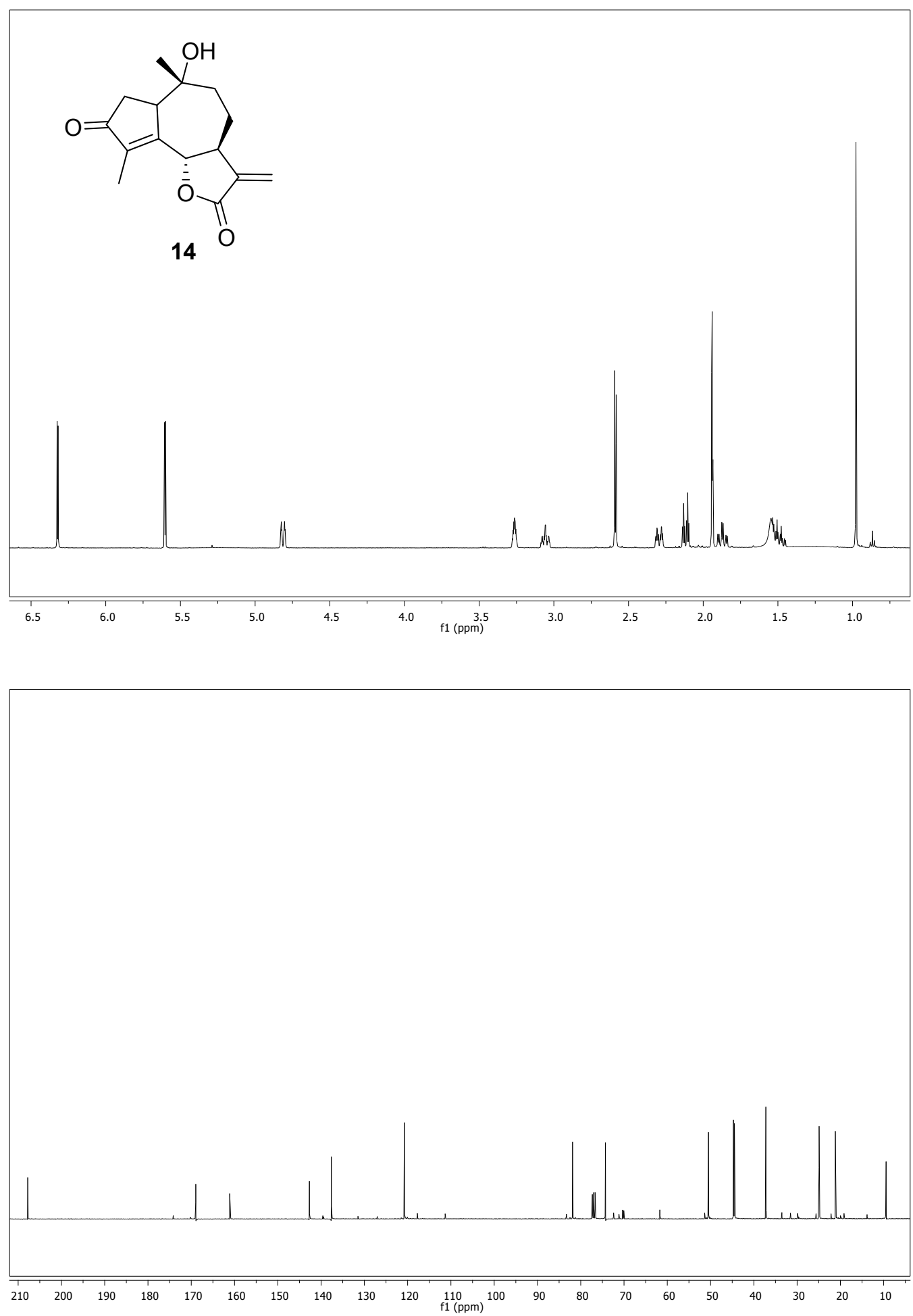
(3S,3aR,6R,9bS)-6-hydroxy-3,6,9-trimethyl-3-(phenylselanyl)-3a, 4,5,6, 6a, 7-

hexahydroazuleno[4,5-b]furan-2,8(3H,9bH)-dione (16).
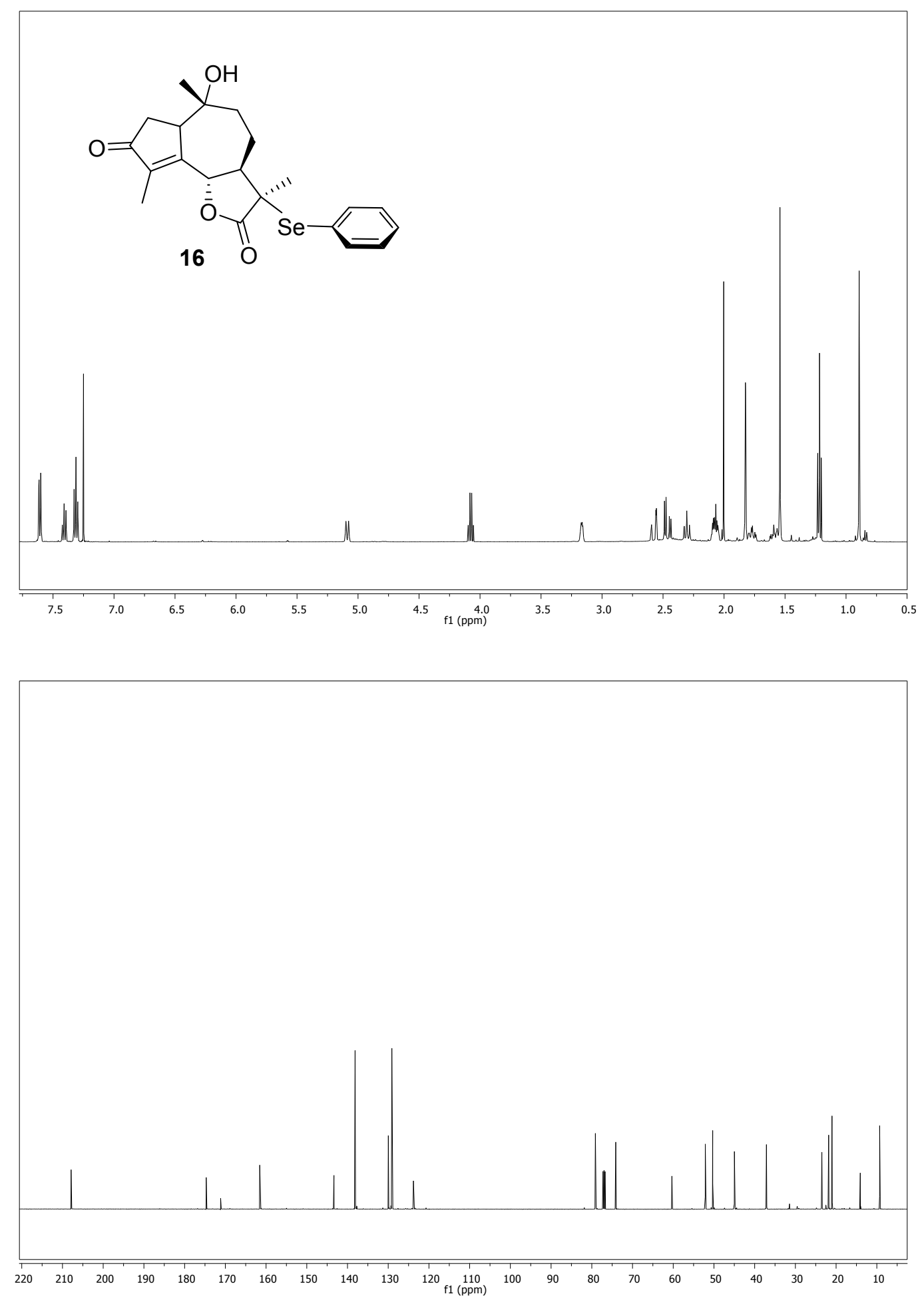

(17).
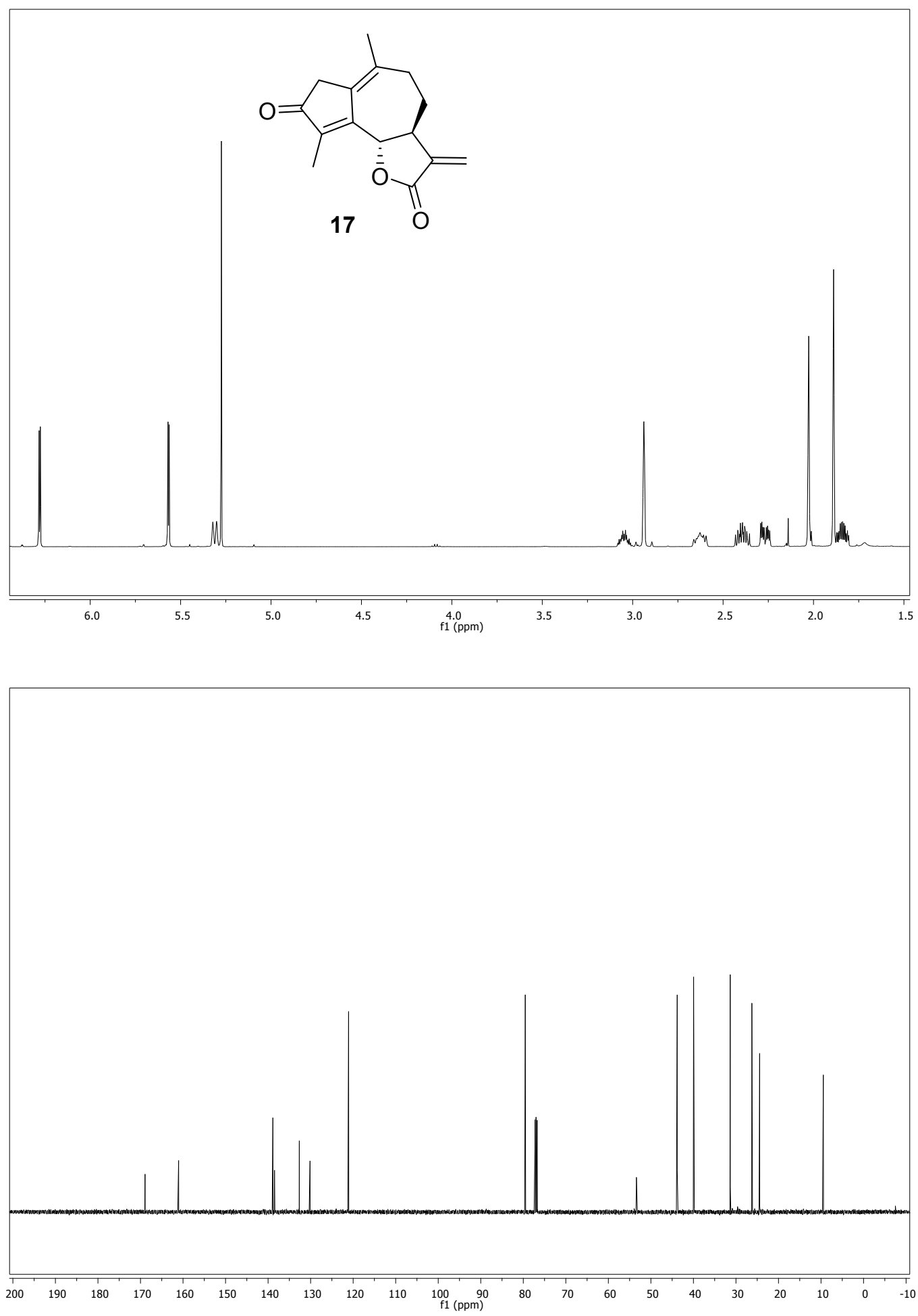

2,8(7H,9bH)-dione (18).
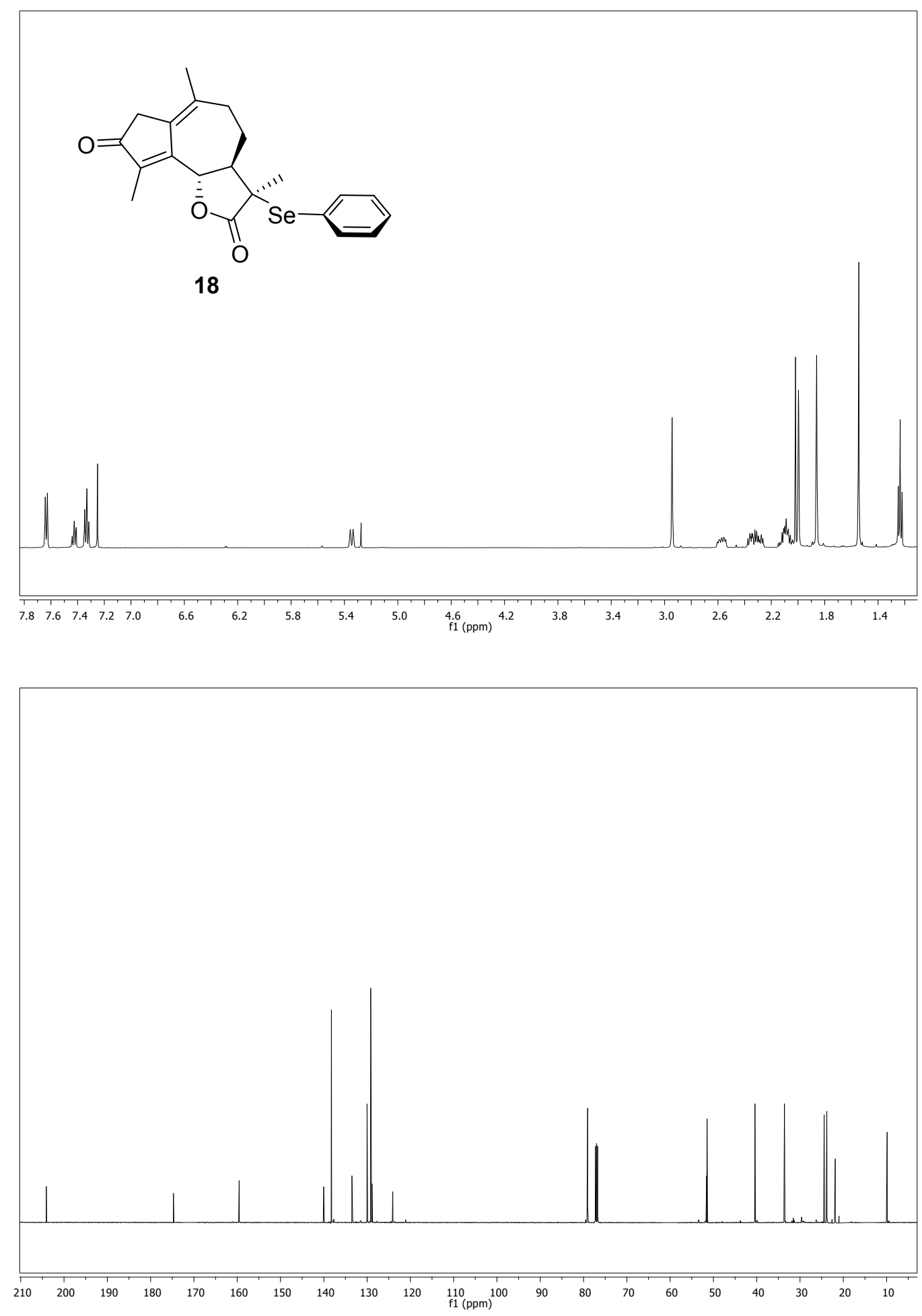
4-methyl-5-((2S,3R,4S)-4-methyl-5-oxo-3-(3-oxobutyl)-4-(phenylselanyl)tetrahydrofuran-2yl)cyclopent-4-ene-1,3-dione (19).
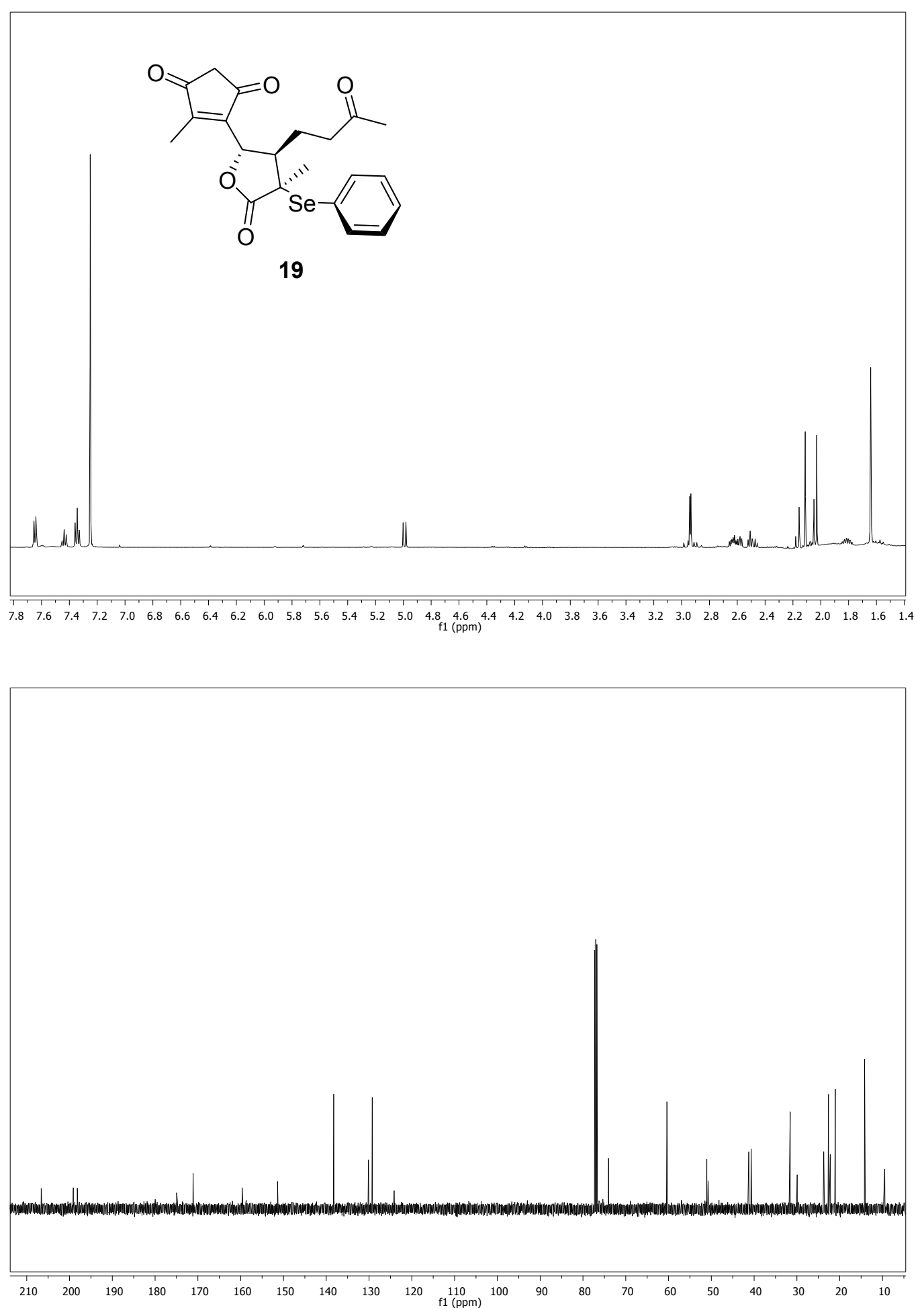
Table S1. $\mathrm{IC}_{50}$ values compounds tested on coleoptile bioassay.

\begin{tabular}{|c|c|c|}
\hline Compound & $\mathbf{I C}_{\mathbf{5 0}}$ & $\mathbf{R}^{\mathbf{2}}$ \\
\hline Logran & 70 & 0.9715 \\
\hline $\mathbf{1 3}$ & 293 & 0.9343 \\
\hline $\mathbf{1 4}$ & 43 & 0.9251 \\
\hline $\mathbf{1 0}$ & 49 & 0.9406 \\
\hline
\end{tabular}


Table S2. $\mathrm{IC}_{50}$ values of compounds tested on STS bioassay

\begin{tabular}{|c|c|c|c|c|c|c|c|c|}
\hline \multicolumn{9}{|c|}{ Root Length } \\
\hline & \multicolumn{2}{|c|}{ Tomato } & \multicolumn{2}{|c|}{ Cress } & \multicolumn{2}{|c|}{ Onion } & \multicolumn{2}{|c|}{ Lettuce } \\
\hline Compounds & $\mathrm{IC}_{50}$ & $\mathbf{R}^{2}$ & $\mathrm{IC}_{50}$ & $\mathbf{R}^{2}$ & $\mathrm{IC}_{50}$ & $\mathbf{R}^{2}$ & $\mathrm{IC}_{50}$ & $\mathbf{R}^{2}$ \\
\hline Logran ${ }^{\circledR}$ & 73 & 0.9720 & 1521 & 0.9676 & 24 & 0.9982 & 2335 & 0.9882 \\
\hline 13 & 125 & 0.9514 & 244 & 0.9309 & 431 & 0.9574 & 1164 & 0.9275 \\
\hline 14 & 995 & 0.9531 & 1278 & 0.9455 & -- & -- & 3340 & 0.902 \\
\hline 10 & 540 & 0.9530 & 370 & 0.9492 & 1491 & 0.9092 & -- & -- \\
\hline \multicolumn{9}{|c|}{ Shoot Length } \\
\hline & \multicolumn{2}{|c|}{ Tomato } & \multicolumn{2}{|c|}{ Cress } & \multicolumn{2}{|c|}{ Onion } & \multicolumn{2}{|c|}{ Lettuce } \\
\hline Compounds & $\mathrm{IC}_{50}$ & $\mathbf{R}^{2}$ & $\mathrm{IC}_{\mathbf{5 0}}$ & $\mathbf{R}^{2}$ & $\mathrm{IC}_{50}$ & $\mathbf{R}^{2}$ & IC $_{50}$ & $\mathbf{R}^{2}$ \\
\hline Logran ${ }^{\circledR}$ & 531 & 0.9231 & 991.5 & 0.9982 & 24 & 0.9355 & 1478 & 0.9442 \\
\hline 13 & 109 & 0.9608 & 430.6 & 0.9574 & 400 & 0.9368 & 8178 & 0.9798 \\
\hline 14 & 2031 & 0.9918 & -- & -- & 2313 & 0.9405 & -- & -- \\
\hline 10 & 722 & 0.9703 & 1491 & 0.9092 & 1014 & 0.945 & 1373 & 0.9062 \\
\hline
\end{tabular}

